\title{
Effects of gallic acid and physical exercise on passive avoidance memory in male rat
}

\author{
Azadeh Salehi' ${ }^{1}$ Zahra Rabiei², Mahbubeh Setorki ${ }^{\circledR 3^{*}}$ \\ ${ }^{I}$ Msc, Department of Biology, Izeh Branch, Islamic Azad University, Izeh, Iran, ${ }^{2}$ Medical Plants Research Center, Basic Health \\ Sciences Institute, Shahrekord University of Medical Sciences, Shahrekord, Iran, ${ }^{3}$ Associate of professor, Department of \\ Biology, Izeh Branch, Islamic Azad University, Izeh, Iran
}

\begin{abstract}
Learning and memory play main roles in daily life of human, and memory represents the basis of all trainings and learning. The aim of the current study is to investigate the effects of gallic acid and physical exercise on the levels of passive avoidance memory in rat. In this experimental study, 46 rats weighing 200-300 g were randomLy divided to six groups of eight each: including control group, groups treated with 10 and $20 \mathrm{mg} / \mathrm{kg}$ gallic acid, group undergoing physical exercise alone, and groups both undergoing physical exercise and treated with 10 and $20 \mathrm{mg} / \mathrm{kg}$ gallic acid. The interventions continued for 10 days. After the intervention, passive avoidance memory was measured by shuttle box, blood samples were taken, and serum and brain antioxidant capacity and malondialdehyde (MDA) levels were measured. Secondary latency in shuttle box significantly increased in groups undergoing treadmill exercise and undergoing treadmill exercise + treating 10 and $20 \mathrm{mg} / \mathrm{kg}$ gallic acid. In groups treated with 10 and $20 \mathrm{mg} / \mathrm{kg}$ gallic acid alone, secondary latency increased significantly. Results confirmed the effects of gallic acid and physical exercise, either alone or combined, in improving memory.
\end{abstract}

Keywords: Gallic Acid/adverse effects. Learning/drug effects. Memory/drug effects. Physical Exercise/ physiology. MDA. Avoidance Learning/drug effects. Exercise/physiology. Rats/physiology. Malondialdehyde/analysis.

\section{INTRODUCTION}

Recent studies have indicated that exercise leads to positive and reinforcing effects on the functions of different organs of the body including the nervous system and brain (Berchtold et al., 2001). For confirming the role of exercise in learning, a study showed that exercise significantly reinforced spatial learning (water maze) of adult male rats (Ahmadiasl, Alaei, Hänninen, 2003).

It seems that exercise boosts memory and learning processes through changes in the chemical composition of the brain (neurotransmitters and nerve growth factors), synaptic plasticity, and neural network connectivity, and strengthening the process of producing neurons on the one hand and strengthening the process of the production of new neurons on the other hand (Park et al., 2017).

Increased antioxidant activity and decreased free radicals due to protein oxidation have optimal effects

\footnotetext{
*Correspondence: Mahbubeh Setorki. Associate of professor, Department of Biology, Izeh Branch, Islamic Azad University, Izeh, Iran. Tel: 00989133121589. Postal code: 8813833435. E-mail: doctor.setorgi@gmail.com
}

on learning and memory (Bhat et al., 2015) and the antioxidant compounds accumulated in the nerve tissue are effective in factors preventing and treating diseases related to oxidative stress. Oxidative stress is considered a risk factor for increasing oxidized lipids and proteins in the central nervous system (CNS) and leads to tissue damage (Bhat et al., 2015). The production of free radicals is associated with normal cell processes such as cell metabolism, mitochondrial breath, and lipoxygenase and cyclooxygenase activity (Kim et al., 2015). Different amounts of the ROS produced in different regions of the brain are likely to be due to the amounts of the consumed energy by these regions, and therefore hippocampus and stratum that consume comparatively larger amounts of oxygen (Kalogeris, Bao, Korthuis, 2014) are more sensitive than other regions. Flavonoids' protective effects depend on their capability of hydrogenating or scavenging free radicals (Ghasemzadeh, Ghasemzadeh, 2011).

Among the new therapeutic strategies to improve cognitive impairments, physical exercise for maintaining the brain health and function has been known to have 
useful effects on learning and long-term memory (Radak et al., 2006).

It seems that gallic acid has antifungal and antibacterial properties. Gallic acid serves as an antioxidant agents and helps the cells of the body be protected against oxidative damage. It has been observed that gallic acid has anticancer property without damaging the cells of the body (Jagan et al., 2008).

Gallic acid is found in drinks, nuts, cucumber, tea leaves, oak bark, strawberry, pineapple, banana, lemon, red grapes, apple skin, and other medicinal herbs. Given its biological activities, gallic acid exerts antibacterial, antiviral, and anti-inflammatory activities, and its antioxidant effects have been demonstrated via inhibiting tyrosinase activities (Aruoma et al., 1993). Gallic acid induces apoptosis in cancer cells and increases glutathione peroxidase (GPX) levels, indicating its antioxidant effects (Lo et al., 2010).

Gallic acid exerts antioxidant effects and our aim is to investigate the effects of exercise, given its optimal effect on memory, and gallic acid, given already confirmed effect of antioxidant compounds in improving memory and learning, both alone and combined on passive avoidance memory in rat.

\section{MATERIAL AND METHOD}

\section{Animals and grouping}

In this study, male Wistar rats weighing 250-300 g were used. The rats were housed under appropriate $\left(21 \pm 2{ }^{\circ} \mathrm{C}\right)$ temperature and cycle of 12-h light:12-h darkness as they had free access to the same water and food.

\section{Exercise (forced running on treadmill)}

To force the rats to run, a rat-specific treadmill was used. The treadmill had a quadrilateral metal body. The rats could run both together and separately on a one-piece conveyor belt. The conveyor belt was located on four rollers with adjustable gradients from 0 to 15 degrees, and a high-powered electric motor with adjustable engine rotation moved the belt (up to speed of $17 \mathrm{~m} / \mathrm{min}$ ). To do treadmill exercise, the rats were initially accustomed to the treadmill one day before the test for half an hour and trained to run it, and the lazy or sick rats were identified and excluded from the study. The main exercise in all steps of the study was the same and was determined to be onehour running per day at speed of $17 \mathrm{~m} / \mathrm{min}$ and surface of 15-degree gradient (Cechetti et al., 2012).

\section{Passive avoidance memory using shuttle box}

To investigate passive avoidance memory, shuttle box was used. This device consists of a light chamber connected to a dark chamber by a guillotine door. Electric shocks are delivered by a separate actuator to the rods of the shuttle box's floor. This test was conducted within four days for each rat. On the first and second days, the rats were left in the box for $5 \mathrm{~min}$ to acclimatize to it.

On the third day, a retention test was conducted. The rats were independently left in the light chamber. After 2-min acclimatization to the light chamber, the guillotine door was opened, and after the rat entered the dark chamber, the guillotine door was closed and an electrical shock ( $1 \mathrm{~mA} /$ second) was exerted to the rat so that it only paddled. In this test, initial latency to enter the dark chamber was recorded. Twenty four hours later, each rat was placed in the light chamber to continue the test. The interval between being placed in the light chamber and entering the dark chamber was considered to indicate step through latency (up to 60 seconds) (Rabiei et al., 2015).

Group 1 (control group): The rats that underwent no physical exercise, received normal saline alone for 10 days, and then underwent shuttle box test;

Group 2: The rats that were intraperitoneally administered with $10 \mathrm{mg} / \mathrm{kg}$ body weight (BW) gallic acid half an hour before the test for 10 days and then underwent shuttle box test within 4 days;

Group 3: The rats that were intraperitoneally administered with $20 \mathrm{mg} / \mathrm{kg} \mathrm{BW}$ and then underwent shuttle box test;

Group 4: The rats that were intraperitoneally administered with $10 \mathrm{mg} / \mathrm{kg}$ BW gallic acid and underwent forced physical exercise at a speed of $17 \mathrm{~m} / \mathrm{min}$ on a surface of 15-degree gradient 1 hour per each day for 10 days, and 1 hour later, they underwent shuttle box test;

Group 5: The rats that were intraperitoneally administered with $20 \mathrm{mg} / \mathrm{kg} \mathrm{BW}$ and underwent forced physical exercise at speed of $17 \mathrm{~m} / \mathrm{min}$ and surface of 15-degree gradient 1 hour per each day for 10 days day, and 1 hour later, the shuttle box test was conducted on them;

Group 6: The rats that underwent forced physical exercise 1 hour per each day for 10 days day at speed of $17 \mathrm{~m} / \mathrm{min}$ and surface of 15 -degree gradient, and 1 hour later, the shuttle box test was conducted on them.

\section{Determining total serum and brain antioxidant capacity using FRAP}

Three solutions were used to measure serum antioxidant capacity consisting of: 1 . Buffer $(1.55 \mathrm{~mL}$ of sodium acetate and $8 \mathrm{~mL}$ of concentrated acetic acid 
reaching $500 \mathrm{~mL}$ volume using distilled water; 2 . Iron chloride solution $(270 \mathrm{mg}$ of iron (III) chloride reaching $50 \mathrm{~mL}$ volume using distilled water; and 3 . Triazine $(47 \mathrm{mg}$ of triazine dissolved in $40 \mathrm{~mL}$ of $40 \mathrm{mM}$ hydrochloric acid). Stock solution was prepared by adding $10 \mathrm{~mL}$ of solution $1,1 \mathrm{~mL}$ of solution 2 , and $1 \mathrm{~mL}$ of solution 3 . $25 \mu \mathrm{L}$ of serum sample or brain homogenate was added to $1.5 \mathrm{~mL}$ of the stock solution and the optical absorption was read at 593-nm wavelength after the stock solution was left at $37^{\circ} \mathrm{C}$ for 10 minutes (Rabiei et al., 2015).

\section{Measuring plasma MDA levels}

Stock solution included $0.5 \mathrm{~g}$ thiobarbituric acid and $80 \mathrm{~mL}$ acetic acid $20 \%$ whose $\mathrm{pH}$ was adjusted to $3.5 \mathrm{using}$ $\mathrm{NaOH}$. The final volume of of the solution was increased to $100 \mathrm{~mL}$ by adding acetic acid $20 \%$. One hundred $\mu \mathrm{L}$ of the solution was dissolved in $100 \mu \mathrm{L}$ SDS $(8.1 \%)$ and $2.5 \mathrm{~mm}$ of the stock solution, and then the tubes were closed with aluminium lids and placed in boiling water bain-marie for 1 hour. Then, the tubes were cooled under the tap water and centrifuged at $4000 \mathrm{rpm}$, and then the optical absorbance of the supernatant was recorded at $532 \mathrm{~nm}$ (Rabiei et al., 2015).

\section{Measuring brain MDA levels}

To measure brain MDA levels, $1 \mathrm{~mL}$ of the homogenized brain tissue (in cooled $2.5 \% \mathrm{KCl}$ at $1: 10$ $\mathrm{w} / \mathrm{v}$ ratio) was poured into a $20-\mathrm{mL}$ glass tube and was incubated in a metabolic shaker at $37 \pm 1{ }^{\circ} \mathrm{C}$ for $60 \mathrm{~min}$. Then, $1 \mathrm{~mL}$ tetrachloroacetic acid $5 \%$ and $1 \mathrm{~mL}$ thiobarbituric acid $67 \%$ were added and mixed well. The content of each vial was centrifuged at $2000 \mathrm{~g}$ for $15 \mathrm{~min}$. Then, supernatant was transferred to another tube and was left in boiling water bath. Then, the tubes were cooled and the optical absorbance of the supernatant was recorded at $532 \mathrm{~nm}$ wavelength (Rabiei et al., 2015).

\section{Statistical analysis}

Data analysis was conducted by SPSS. First, normal distribution of the data was investigated by KolmogorovSmirnov test and variance homogeneity was studied by Levene's test. Then, to investigate the significance of difference between the treatments, one-way ANOVA was used, and to compare the mean values, Tukey's test. The data were expressed as mean (standard deviation) and $p<0.05$ was considered statistically significant.

\section{RESULTS}

Figure 1 illustrate the effects of gallic acid and physical exercise, alone and combined, on memory and passive avoidance learning. There was no significant difference in initial latency among the groups. Results showed that the secondary latency in shuttle box increased significantly in the groups undergoing treadmill and undergoing treadmill + receiving 10 and $20 \mathrm{mg}$ gallic acid compared to the control group. The secondary latency increased significantly in the groups that received gallic acid alone compared to the control group.

\section{shuttle box}

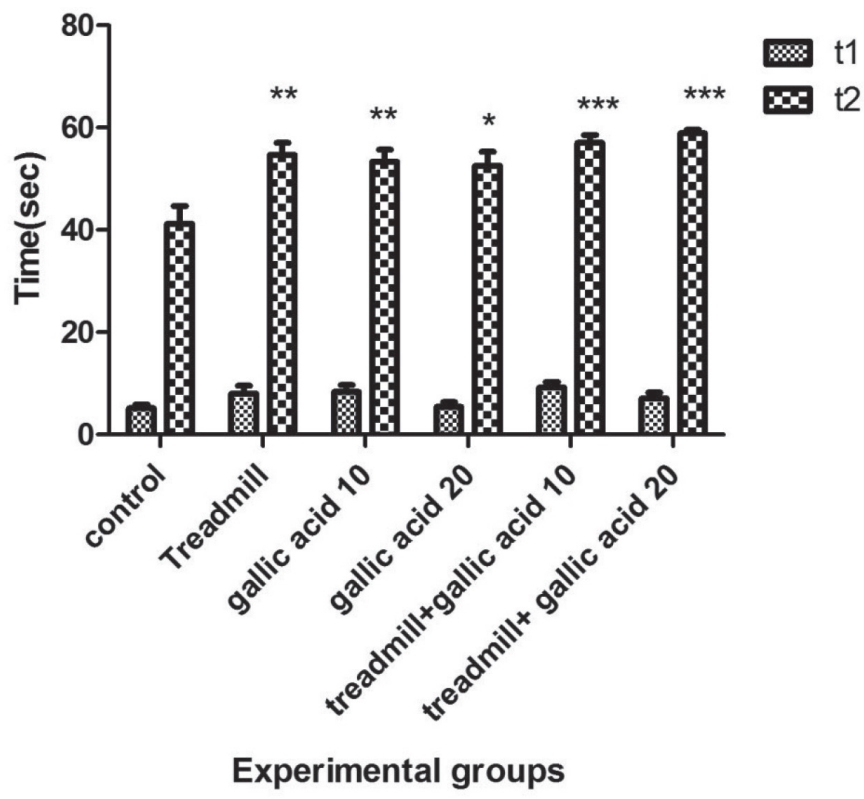

FIGURE 1 - The effects of gallic acid and physical exercise, alone and combined, on initial latency and secondary latency in passive avoidance memory test. $*=(p<0.05) ; * *=(p<0.01)$; $* * *=(p<0.001)$.

As Figure 2 illustrates, serum antioxidant level increased significantly in the group that received $20 \mathrm{mg} / \mathrm{kg}$ gallic acid compared to control group, and in the groups undergoing treadmill exercise + receiving gallic acid (10 and $20 \mathrm{mg} / \mathrm{kg}$ ) compared to control group. There was no significant difference in serum antioxidant level between exercise group and the group that received $10 \mathrm{mg} / \mathrm{kg}$ gallic acid alone, and control group.

As Figure 3 illustrates, brain antioxidant capacity increased significantly in the group undergoing treadmill exercise + receiving $20 \mathrm{mg} / \mathrm{kg}$ gallic acid compared to control group, but there was no significant difference in brain antioxidant capacity between the other groups and control group.

As Figure 4 illustrates, serm MDA level decreased significantly in the group that received $20 \mathrm{mg} / \mathrm{kg}$ gallic acid compared to control group. In addition, serum MDA 
Frap serum

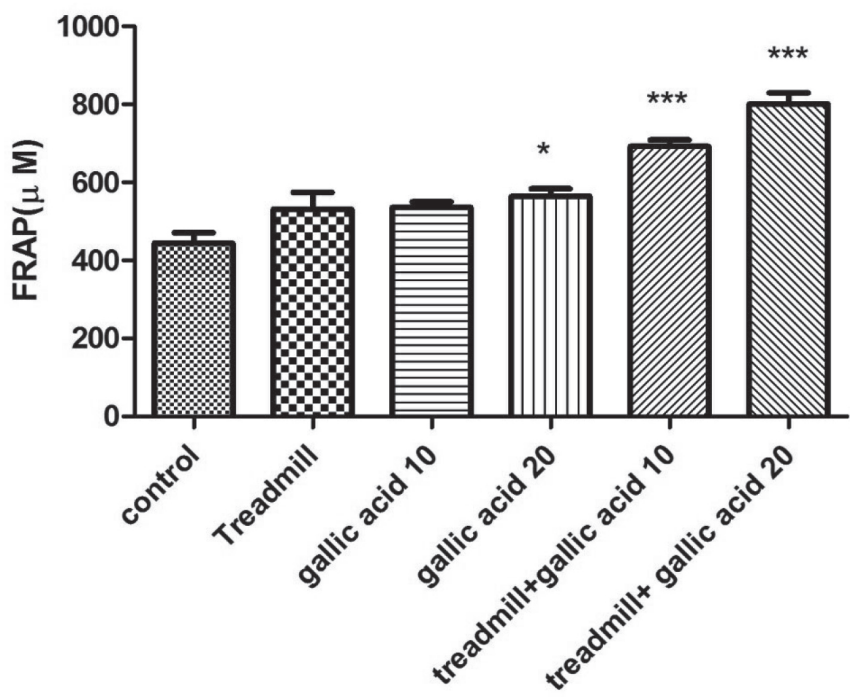

Experimental groups

FIGURE 2 - The effects of gallic acid and physical exercise, alone and combined, on serum antioxidant capacity; $*=(p<0.05)$; $* * *(p<0.001)$.

\section{Frap brain}

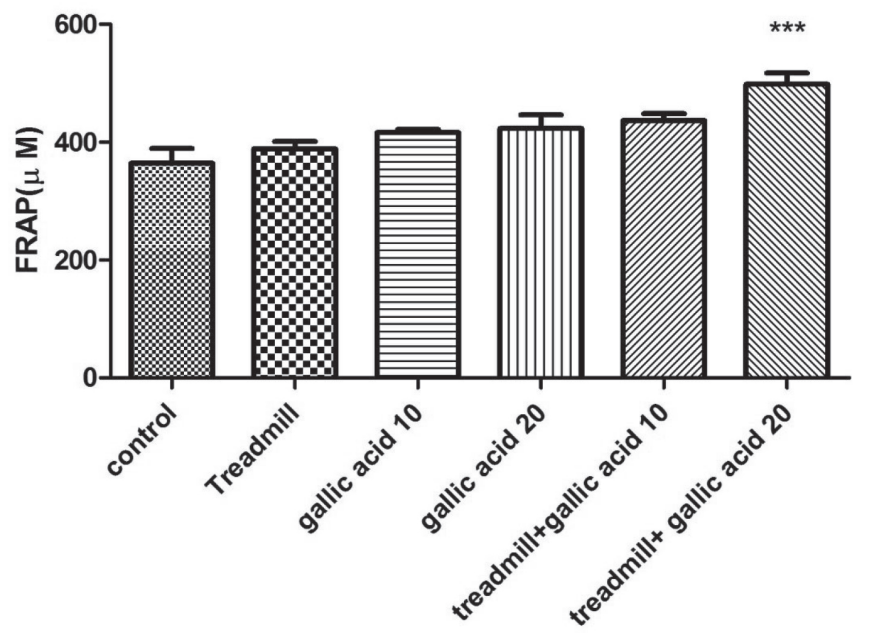

Experimental groups

FIGURE 3 - The effects of gallic acid and physical exercise, alone and combined, on brain antioxidant capacity; $* * *=(p<0.001)$.

level decreased significantly in the groups undergoing treadmill exercise + receiving gallic acid (10 and $20 \mathrm{mg} / \mathrm{kg}$ ) compared to the control group. Serum MDA level decreased significantly in treadmill exercise group and the groups treated with gallic acid (10 and $20 \mathrm{mg} / \mathrm{kg}$ ) alone compared to control group yet insignificantly.

As Figure 5 illustrates, brain MDA level decreased significantly in the group undergoing treadmill exercise compared to control group. Besides that, brain MDA level decreased significantly in the groups receiving gallic serum MDA

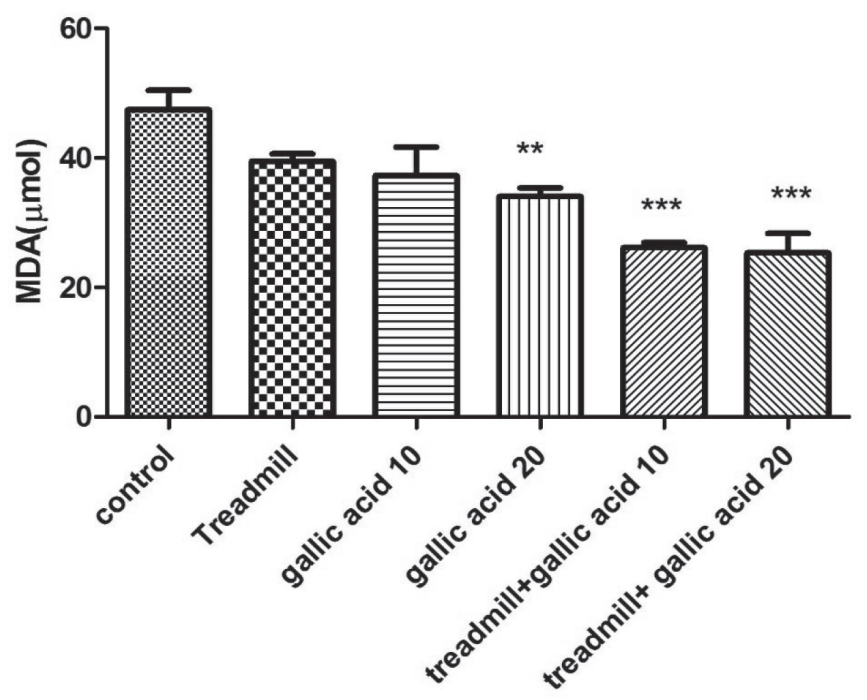

Experimental groups

FIGURE 4 - The effects of gallic acid and physical exercise, alone and combined, on serum malondialdehyde level; $* *=(p<0.01) ; * * *=(p<0.001)$.

\section{brain MDA}

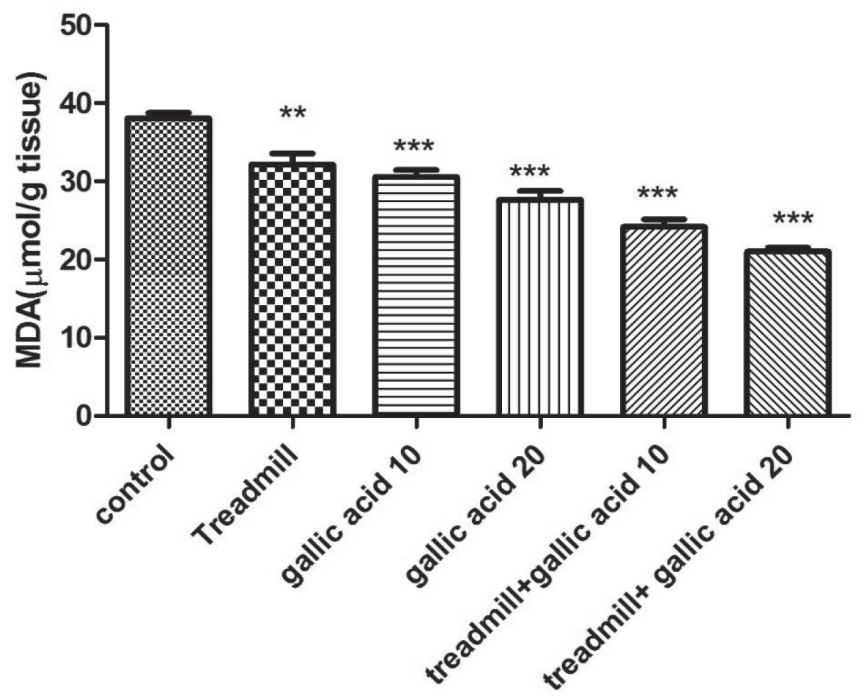

Experimental groups

FIGURE 5 - The effects of gallic acid and physical exercise, alone and combined, on brain antioxidant level; $* *=(p<0.01)$; $* * *=(p<0.001)$.

acid (10 and $20 \mathrm{mg} / \mathrm{kg}$ ) alone and the groups undergoing treadmill exercise + receiving gallic acid (10 and $20 \mathrm{mg} / \mathrm{kg}$ ) compared to control group.

\section{DISCUSSION}

Results showed that gallic acid either alone or 
combined with physical exercise improved learning and memory in passive avoidance memory.

Regarding the mechanisms of memory and learning, much evidence has indicated that the amygdala and GABAergic system in different models are involved in memory modulation and storage. The presence of GABAergic receptors in the basolateral amygdala raises the probability of their role in memory storage in this region. Rashidy-Pour et al. (2015) studied the role of the GABAergic system in acquiring, consolidating, and retrieving the newly learned information in the spatial avoidance learning model, and observed that parenteral GABA agonist (muscimol) significantly impaired the storage and retrieval of spatial memory (Rashidy-Pour, Vafaei, 2001). (Mansouri et al. 2014) studied the potential mechanism of gallic acid anxiolytic effects in rat using plus maze test. Gallic acid exerts anxiolytic effect in low doses and sedative effect in high doses that is comparable to diazepam ( $1 \mathrm{mg} / \mathrm{kg}$, i.p.) and buspirone $(1 \mathrm{mg} / \mathrm{kg}$, i.p.). Pretreatment with flumazenil, as a benzodiazepine antagonist, blocks anxiolytic effects of gallic acid. The anxiolytic effects of gallic acid are strongly blocked by WAY 100635 of the HT1A-5 antagonist receptor (Mansouri et al., 2014).

Pharmacological studies on human subjects have indicated that inhibition of muscarinic cholinergic receptors by certain drugs such as scopolamine disrupts the recording of new memories, but does not affect the retrieval of previous memories. In addition, it has been reported that the drugs that activate nicotinic receptors are effective in encoding new memories. Studies have shown that acetylcholine can increase the LTP process in many regions of the brain, including the hippocampus, the entorhinal cortex, and the piriform cortex (Hasselmo, 2006). Gallic acid exerts strong acetylcholinesterase property in a dose-dependent manner with $\mathrm{IC}_{50}$ of 138.5 $\pm 0.25-397.6 \pm 5.2 \mu \mathrm{g} / \mathrm{mL}$ (Cho et al., 2011). Given that the inhibition of acetylcholinesterase by gallic acid has been confirmed, it seems that prevention of memory and learning loss by gallic acid is associated with inhibition of acetylcholinesterase and therefore increased levels of acetylcholine in the brain tissue (Mansouri et al., 2013)

Different studies have indicated that with increasing age, increased production of reactive oxygen species (ROS) and oxidative stress results in the brain dysfunction and the loss of memory and learning. Oxidative stress is considered to be a risk factor for increased oxidizing lipids and proteins in the CNS and therefore tissue damage (Alibabaei et al., 2014). Different amounts of the ROS produced in different regions of the brain may be related to the amount of energy consumed in these regions, and therefore the hippocampus and stratum that consume comparatively larger amounts of energy are more sensitive than other regions of the brain (Alibabaei et al., 2014). Memory-boosting effects of gallic acid are likely to be due to its antioxidant property and therefore its prevention of oxidative stress in the brain tissue.

Gallic acid has potential to exert inhibitory effect against the neurotoxicity due to NMDA receptors sensitivity and glutamate-induced toxicity following cerebral ischemia that lead to calcium entry into the cell, intracellular calcium accumulation, and finally apoptosis. In other words, gallic acid prevents activation of the NMDA receptors through its antioxidant property and exerts protective effects against ischemia (Korani et al., 2014).

A number of studies have shown the optimal effects of physical exercise on memory and learning. During exercise, endorphin is released that tends to focus more on the brain's priorities and maintain them further. Indeed, the ability of the brain to focus on mental priorities for longer term can be improved (Veening, Barendregt, 2015). Catecholamines are a group of chemical compounds including norepinephrine, epinephrine, and dopamine that serve as chemical transmitters. Norepinephrine and dopamine contribute to learning. Regular exercise increases the secretion and plasma levels of these compounds. Therefore, doing regular exercise can improve memory via enhancing secretion of chemical transmitters (Veening, Barendregt, 2015). A study showed that exercise caused increase in the number of new hippocampal cells and improvement of brain function (Biedermann et al., 2016).

Besides that, physiological investigations have shown that physical movements increase hippocampal electrical activity, which may be due to changes in neuronal activity and neurotransmitters (Voss et al., 2013). A study on aerobic exercise showed that exercise improved spatial memory and hippocampal neuronal density in the DG and other regions of the hippocampus without changing apoptosis rate, and therefore short-term memory (Mireku, Power, Thompson, 2013). Researchers argue that exercise may reinforce the hippocampal structure in human (Snow, Mang et al., 2016). Exercise leads to increased levels of nutrophen that can be a cause of hippocampal volume. Nutrophen enhances the viability and regeneration of the neurons, causes enhancement, stimulation, and growth of these cells, and is essential for memorization and memory (Maass et al., 2016). Neurotrophic factors such as brainderived neurotrophic factor (BDNF), nerve growth factor, and fibroblast growth factor contribute to support and growth of different types of cerebral neurons (RashidyPour, Fathollahi et al., 2015). 
It has also been reported that exercise can indirectly influence expression of neurotrophic factors genes through affecting secretion of neurotransmitters such as acetylcholine, gaba-aminobutyric acid, and monoamines. BDNF is a protein that is encoded by BDN gene. This factor is from family neurotrophins that helps to expand neural network. BDNF is one of the most important members of this family and causes launching of intracelluar cascades and eventually production and differentiation of new neurons (Rashidy-Pour, Fathollahi et al., 2015). This factor serves as a mediator of synaptic effects, neural bindings, and plasticity in the brain.

\section{CONCLUSION}

The findings of the current study confirm the memory-boosting effects of gallic acid and physical exercise, either alone and combined.

\section{ACKNOWLEDGMENTS}

The authors thanks of laboratory Islamic Azad University Izeh.

\section{COMPETING INTERESTS} interests.

The authors declare that they have no competing

\section{REFERENCES}

Ahmadiasl N, Alaei H, Hänninen O. Effect of exercise on learning, memory and levels of epinephrine in rats' hippocampus. J Sports Sci Med, 2003;2(3):106-9.

Alibabaei Z, Rabiei Z, Rahnama S, Mokhtari S, Rafieian-kopaei M. Matricaria Chamomilla extract demonstrates antioxidant properties against elevated rat brain oxidative status induced by amnestic dose of scopolamine. Biomed Aging Pathol. 2014;4(4):355-60.

Aruoma OI, Murcia A, Butler J, Halliwell B. Evaluation of the antioxidant and prooxidant actions of gallic acid and its derivatives. J Agric Food Chem. 1993; 41(11):1880-5.

Bhat AH, Dar KB, Anees S, Zargar MA, Masood A, Sofi $\mathrm{MA}$, et al. Oxidative stress, mitochondrial dysfunction and neurodegenerative diseases; a mechanistic insight. Biomed Pharmacother. 2015;74:101-10.
Berchtold NC, Kesslak JP, Pike CJ, Adlard PA, Cotman $\mathrm{CW}$. Estrogen and exercise interact to regulate brain derived neurotrophic factor mRNA and protein expression in the hippocampus. Eur J Neurosci.2001;14(12):1992-2002.

Biedermann SV, Fuss J, Steinle J, Auer MK, Dormann C, FalfánMelgoza C, et al. The hippocampus and exercise: histological correlates of MR-detected volume changes. Brain Struct Func. 2016;221(3):1353-63.

Cechetti F, Worm PV, Elsner VR, Bertoldi K, Sanches E, Ben $\mathrm{J}$, et al. Forced treadmill exercise prevents oxidative stress and memory deficits following chronic cerebral hypoperfusion in the rat. Neurobiol Learn Mem. 2012;97(1):90-6.

Cho Y-S, Kim S-K, Ahn C-B, Je J-Y. Inhibition of acetylcholinesterase by gallic acid-grafted-chitosans. Carbohydr Polym. 2011; 84(1):690-3.

Ghasemzadeh A, Ghasemzadeh N. Flavonoids and phenolic acids: Role and biochemical activity in plants and human. J Med Plant Res. 2011;5(31):6697-703.

Hasselmo ME. The role of acetylcholine in learning and memory. Curr Opin Neurobiol. 2006;16(6):710-5.

Jagan S, Ramakrishnan G, Anandakumar P, Kamaraj S, Devaki T. Antiproliferative potential of gallic acid against diethylnitrosamine-induced rat hepatocellular carcinoma. Mol Cell Biochem. 2008;319(1/2):51-9.

Kalogeris T, Bao Y, Korthuis RJ. Mitochondrial reactive oxygen species: a double edged sword in ischemia/reperfusion vs preconditioning. Redox Biol. 2014;2:702-14.

Kim GH, Kim JE, Rhie SJ, Yoon S. The role of oxidative stress in neurodegenerative diseases. Exp Neurobiol. 2015;24(4):325-40.

Korani MS, Farbood Y, Sarkaki A, Moghaddam HF, Mansouri MT. Protective effects of gallic acid against chronic cerebral hypoperfusion-induced cognitive deficit and brain oxidative damage in rats. Eur J Pharmacol. 2014; 733:62-7.

Lo C, Lai T-Y, Yang J-H, Yang J-S, Ma Y-S, Weng S-W, et al. Gallic acid induces apoptosis in A375. S2 human melanoma cells through caspase-dependent and-independent pathways. Int J Oncol. 2010;37(2):377-85. 
Maass A, Düzel S, Brigadski T, Goerke M, Becke A, Sobieray U, et al. Relationships of peripheral IGF-1, VEGF and BDNF levels to exercise-related changes in memory, hippocampal perfusion and volumes in older adults. Neuroimage. 2016:131:142-54.

Mansouri MT, Naghizadeh B, Ghorbanzadeh B, Farbood Y, Sarkaki A, Bavarsad K. Gallic acid prevents memory deficits and oxidative stress induced by intracerebroventricular injection of streptozotocin in rats. Pharmacol Biochem Behav. 2013;111:906.

Mansouri MT, Soltani M, Naghizadeh B, Farbood Y, Mashak A, Sarkaki A. A possible mechanism for the anxiolytic-like effect of gallic acid in the rat elevated plus maze. Pharmacol Biochem Behav. 2014;117:40-6.

Park E, Park K, Liu E, Jiang R, Zhang J, Baker AJ. Bonemarrow-derived endothelial progenitor cell treatment in a model of lateral fluid percussion injury in rats: evaluation of acute and subacute outcome measures. J Neurotrauma. 2017;34(19):280111 .

Rabiei Z, Mokhtari S, Asgharzade S, Gholami M, Rahnama S, Rafieian-kopaei M. Inhibitory effect of Thymus vulgaris extract on memory impairment induced by scopolamine in rat. Asian Pac J Trop Biomed. 2015;5(10):845-51.
Radak Z, Toldy A, Szabo Z, Siamilis S, Nyakas C, Silye G, et al. The effects of training and detraining on memory, neurotrophins and oxidative stress markers in rat brain. Neurochem Int. 2006;49(4):387-92.

Rashidy-Pour A, Fathollahi Y, Miladi-Gorji H, Safari M. Enhancing hippocampal neuronal numbers in morphinedependent rats by voluntary exercise through a brain-derived neurotrophic factor-mediated mechanism. Middle East J Rehabil Health. 2015;2(1):1-5.

Rashidy-Pour A, Vafaei A. The effect s of muscimol injection into basolateral amygdala on spatial memory processing in place avoidance learning task. Koomesh. 2001;2(2):73-81.

Snow NJ, Mang CS, Roig M, McDonnell MN, Campbell KL, Boyd LA. The effect of an acute bout of moderate-intensity aerobic exercise on motor learning of a continuous tracking task. PloS one. 2016;11(2):e0150039.

Veening JG, Barendregt HP. The effects of Beta-endorphin: state change modification. Fluids Barriers CNS. 2015;12(3):1-22.

Voss MW, Vivar C, Kramer AF, van Praag H. Bridging animal and human models of exercise-induced brain plasticity. Trends Cogn Sci. 2013;17(10):525-44.

Received for publication on $07^{\text {th }}$ April 2018 Accepted for publication on $16^{\text {th }}$ May 2018 